\title{
Tic douloureux
}

\author{
John D Loeser MD
}

\section{JD Loeser. \\ Tic douloureux. \\ Pain Res Manage 2001;6(3):156-165.}

Tic douloureux is an excruciatingly painful condition that primarily affects elderly people. It consists of unilateral electric shocklike facial pains triggered by non-noxious stimulation with clear-cut pain-free intervals. It should be discriminated from all other types of facial pain by the history and physical examination. Primary treatment includes anticonvulsant drugs; if these fail or side effects prevent their use, a surgical procedure is warranted. Almost every patient with tic douloureux can be relieved of his or her pain with anticonvulsant medications or surgery. Stereotactic radiosurgery, percutaneous gangliolysis and suboccipital craniectomy with microvascular decompression are the primary surgical options. The common aspects of tic douloureux and some of the rarer variations are reviewed, and treatment options are presented.

Key Words: Anticonvulsants; Facial pain; Pain; Pain surgery; Tic douloureux; Trigeminal neuralgia

\begin{abstract}
Tic douloureux
RÉSUMÉ : Le tic douloureux est un syndrome extrêmement souffrant, qui touche surtout les personnes âgées. Il consiste en des crises faciales unilatérales, qui ressemblent à des décharges électriques, déclenchées par des stimulus inoffensifs et entrecoupées de période de repos nettement définies. On devrait distinguer le tic douloureux de toutes les autres formes de douleur faciale par l'anamnèse et un examen physique. Les anticonvulsivants constituent le traitement de première intention mais, en cas d'échec ou d'apparition d'effets indésirables, l'intervention chirurgicale est justifiée. Presque tous les cas de tic douloureux cèdent au traitement anticonvulsivant ou à la chirurgie. Figurent parmi les principales options thérapeutiques la chirurgie stéréotaxique, la gangliolyse percutanée et la craniectomie sous-occipitale suivie d'une décompression vasculaire microchirurgicale. Il sera ici question des formes courantes et des formes plus rares de tic douloureux ainsi que des différentes options thérapeutiques.
\end{abstract}

$\mathrm{E}$ very physician should be able to establish the diagnosis of tic douloureux or classic trigeminal neuralgia, because this terrible pain syndrome can almost always be controlled by medication or surgery (1). Other types of facial pain do not respond often to either the medicines or the surgery that are so effective for tic. Some pains are not reduced by drugs or may be made worse by the surgeries that dramatically relieve the pain of tic. Furthermore, treating sinuses, teeth or temporomandibular joints does not alleviate tic.

The first written description of tic has been ascribed to Johannes Bausch in 1672. André coined the term 'tic douloureux' in 1756, and Fothergill provided a vivid description of this pain syndrome in 1773. Early medical therapies were not efficacious; the first useful treatment was probably trichloroethylene inhalation, which was initiated in the 1920 s. It is believed that the 16th century stone carvings in Wells Cathedral depict the pain of trigeminal neuralgia (2). Maréschal, surgeon to King Louis XIV of France, cut the peripheral branches of the trigeminal nerve for pain (2). Effective surgical procedures that injured the trigeminal nerve were developed in the early 18 th century. A major step forward in 1891 was Sir Victor Horsley's subtemporal retrogasserian neurotomy. Both Hartley and Krause developed similar operative approaches, and in 1900 Harvey Cushing described a simplified approach. Suboccipital retrogasserian neurotomy was developed by Fraser in 1901 and popular- 
ized by Dandy in the next three decades; Jannetta developed suboccipital craniectomy with microvascular decompression in the early 1970 s. Gangliolysis became popular shortly thereafter. Stereotactic radiosurgery, developed by Leksell, became widely available in the 1990s and ushered in a totally new method of relieving tic pain. Neurosurgical treatments for facial pain have been reviewed by Walker and others $(3,4)$.

\section{EPIDEMIOLOGY}

Tic douloureux is predominantly a disease of elderly people, but it can occur uncommonly in young adults (Figure 1). The most common incidence is between the ages 50 and 70 years. About $60 \%$ of patients are female, although publications in the first half of the 20th century described a much higher preponderance of women (5). An early onset of tic douloureux is rarely a sign of multiple sclerosis; patients with only tic should never be told that they have this disease. There are no known geographic, racial or ethnic factors in the incidence of tic douloureux.

\section{ETIOLOGY}

The observations made by Dandy (5), and by Jannetta (6) and other neurological surgeons since then have made it clear that the vast majority of patients with tic douloureux have mechanical compression of the trigeminal nerve as it leaves the pons and travels across the subarachnoid space to Meckel's cave. The most common finding is cross compression by a major artery, usually the superior cerebellar artery. A few patients have been found to have veins running across or even through the nerve, and a small incidence of arteriovenous malformation or tumours has also been reported.

The region of impingement on the trigeminal root usually correlates with the location of facial pain. When the pain is perceived in the second or third trigeminal division, compression of the rostral and anterior portion of the nerve by the superior cerebellar artery is common. If first division pain is present, the most frequent finding is compression of the root in its caudal and posterior portion, most often by the anterior inferior cerebellar artery.

A small percentage of patients with tic douloureux have multiple sclerosis as an underlying disease. The autopsy findings on these patients, which have been confirmed by a small number of surgical observations, include a demyelinating plaque in the trigeminal posterior root (7). Plaques also are frequently found in the descending trigeminal tract and in the lemniscal systems; it is not possible to argue that a plaque in the trigeminal root is either necessary or sufficient to cause tic douloureux.

Another theory has been described in the dental literature. Ratner et al (8), and Shaber and Krol (9) have claimed that tic douloureux is caused by foci of abscess and bone resorption with irritation of the trigeminal nerve in the maxilla or mandible, but this theory is not widely accepted, even among dentists. Similar cavities are seen in the jaws of people with no pain or history of pain. The treatment of such

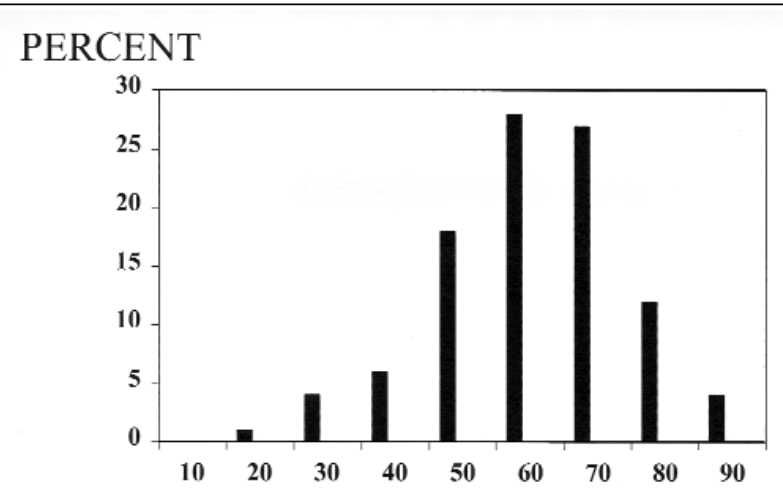

AGE AT DIAGNOSIS

Figure 1) Age of onset of tic douloureux

cavities does not uniformly lead to symptom abatement. Some patients with tic douloureux cannot be shown to have these cavities, yet this concept persists in the dental literature $(10,11)$. Dentists also are fixated on peripheral lesions as the necessary conditions for pain; hence, patients with tic douloureux are subjected to root canal procedures and extractions of normal teeth in the forlorn hope that the pain will cease if the offending tooth or its nerve is removed. Some patients even undergo multiple extractions, only to realize that there was nothing wrong where it hurt, because the pain persists although the tooth is gone.

\section{PATHOPHYSIOLOGY}

How trigeminal nerve pathology can cause a unique pain such as tic douloureux is unclear. No animal model for tic has thus far been developed. Two broad schemes for the explanation of tic douloureux can be identified: 'centralist' and 'peripheralist'. The former scheme is based on the similarities of tic douloureux to focal epilepsy and emphasizes the role of deafferentation in the genesis of neuronal hyperactivity (12). Epileptogenic agents injected into the trigeminal nucleus can cause neuronal hyperactivity and a pain syndrome in cats and monkeys (13). This pain is, however, continuous and does not appear, on the basis of the animal's behaviour, to be intermittent and explosive. Changes in dorsal root reflexes and synaptic function in the trigeminal system have also been described (14).

The peripheralist concept notes that changes in the trigeminal nerve myelin and axons can lead to altered peripheral nerve sensitivity to chemical and mechanical stimuli, and ties the pain syndrome to suspected peripheral causes (15-18). However, many of the purported etiological changes in the peripheral nerve and Gasserian ganglion are related to age and not to the presence or absence of pain. Theories based on ephaptic connections, reverberating circuits and altered central connectivity caused by deafferentation have also been proposed $(19,20)$.

Calvin and colleagues (21) concluded that both peripheral and central mechanisms were required for the produc- 
TABLE 1

Neuropathic facial pain

\begin{tabular}{|c|c|c|c|}
\hline \multirow[b]{2}{*}{ Feature } & \multirow[b]{2}{*}{$\begin{array}{l}\text { Typical neuralgia } \\
\text { (tic douloureux) }\end{array}$} & \multicolumn{2}{|c|}{ Atypical neuralgia } \\
\hline & & $\begin{array}{l}\text { Unilateral } \\
\text { facial pain }\end{array}$ & $\begin{array}{c}\text { Bilateral } \\
\text { facial pain }\end{array}$ \\
\hline Frequency & $\begin{array}{l}\text { Intermittent: every few moments } \\
\text { to once a day or even less }\end{array}$ & Constant, can fluctuate & $\begin{array}{l}\text { Constant, not } \\
\text { much variation }\end{array}$ \\
\hline Pain-free intervals & Always & Rarely & Never \\
\hline Description & Electric shock, stabbing, shooting & $\begin{array}{l}\text { Burning, aching, can have } \\
\text { superimposed shocks }\end{array}$ & Burning, aching \\
\hline Location: nervus intermedius & $\begin{array}{l}\text { Unilateral, usually trigeminal, } \\
\text { glossopharyngeal or vagal }\end{array}$ & Trigeminal or upper cervical often & Usually perioral, rarely intraoral \\
\hline Sensory changes & None or mild hypesthesia & Often hypesthesia & $\begin{array}{c}\text { Common hypesthesia, dysesthesia, } \\
\text { paresthesia }\end{array}$ \\
\hline Precipitating factor & $\begin{array}{l}\text { Triggered by non-noxious stimulation, } \\
\text { often in anterior face and remote from pain }\end{array}$ & $\begin{array}{l}\text { Rarely triggered; trigger } \\
\text { usually in the area of pain }\end{array}$ & Not triggered \\
\hline Autonomic changes & None & Rarely present & None \\
\hline Local tenderness & None & Rare & Rare \\
\hline Causative factors & $\begin{array}{l}\text { Vascular compression of nerve in } \\
\text { subarachnoid space; rarely multiple sclerosis }\end{array}$ & $\begin{array}{l}\text { Tumour, infection, trauma or } \\
\text { mechanical impingement on } \\
\text { nerve; often none found }\end{array}$ & None known \\
\hline Common age at onset (years) & Over 50 & 30 & Over 45 \\
\hline Sex & $60 \%$ female & $75 \%$ female & $90 \%$ female \\
\hline
\end{tabular}

tion of tic douloureux. Fromm and associates (22) proposed a similar mechanism - that a peripheral nerve lesion (in the trigeminal root or distal) was the first event in a process that led to central synaptic changes. The response of the central synapses to altered peripheral events leads to the development of tic douloureux. Complex theories are required to explain the phenomena of tic douloureux: triggering by non-noxious stimuli; separation of the trigger area from the painful region; sudden onset and cessation of pain; and the response to anticonvulsant medications. The new molecular biology of pain has not yet led to an understanding of the mechanisms of tic douloureux.

\section{SYMPTOMS AND SIGNS}

Tic douloureux is characterized by electric shock-like stabbing pains; unilateral pain during any one episode; abrupt onset and termination of pain; pain-free intervals between attacks; non-noxious stimulation triggering the pain, which is often in a different area of the face; minimal or no sensory loss in the region of pain; and pain restricted to the trigeminal nerve (Table 1) (1). Deviations from this typical picture can occur, but the more unusual features that the patient manifests, the less likely that the response to either medication or surgery will be favourable. A history of bilateral tic pain can be elicited in approximately $3 \%$ of patients. Rarely does a patient have bilateral tic pain during one episode; usually an interval of years separates the occurrence of pains on the two sides. I have seen only one patient having tic pains on both sides of his face in the same epoch.

Surgical or anesthetic procedures that damage the trigeminal nerve can produce changes in the findings and in the patient's symptoms. It is essential to ascertain what the symptoms were before any operation. Nerve damage can lead to a burning component of the pain that is uncommon in tic patients without superimposed nerve injury. Significant sensory loss mandates a thorough search for structural pathology such as tumour or infection that is damaging the trigeminal nerve. However, careful sensory testing, especially in patients with long standing tic, often reveals a subtle decrease in light touch and pin-prick sensation in the region of pain in the absence of any prior surgical procedure, neoplasm or infection. This sensory loss is usually not apparent to the patient until it is formally tested.

Most patients with tic douloureux describe a specific triggering stimulus - touching the face, chewing, talking or swallowing. Patients frequently report that exposure of their face to cold triggers pain. The cutaneous trigger is always a non-noxious stimulus and is usually located in the anterior face (19). The trigger area is always ipsilateral to the pain but can be in the same or a different division of the trigeminal nerve. Rarely, the patient reports a trigger area outside the trigeminal territory, usually in the upper cervical dermatomes. I have seen one patient who had a hemispheral infarction and resultant hemiplegia and hemisensory deficit whose pre-existing tic pain was triggered by stimulating his thumb and index finger ipsilateral to his facial pain. Patients with triggering from the scalp often refuse to wash or brush their hair; shaving can be impossible for a man with triggering from the upper lip or face, and oral hygiene is often impossible when the triggering is from the teeth or gingivae. When swallowing or chewing triggers the pain, the patient may develop inadequate caloric intake and dehydration. 


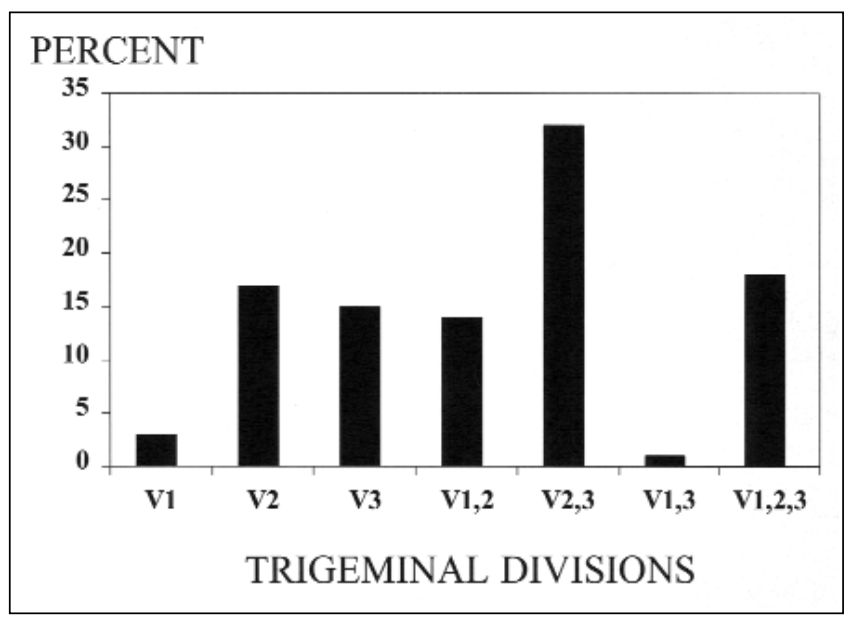

Figure 2) Distribution of pain among divisions of the trigeminal nerve in patients with tic douloureux

In the overwhelming majority of patients with tic douloureux, the pain is restricted to the trigeminal dermatomes. A small number have pain both in the trigeminal areas and in the territory of the nervus intermedius (cranial nerve VII), glossopharyngeal nerve (cranial nerve IX) or vagus nerve (cranial nerve $X$ ) (23). The pains of tic douloureux can also be experienced exclusively in one of these areas (see below). The most common site of tic pain is the combination of the second and third divisions; the rarest site is the combination of the first and third divisions. Figure 2 depicts the patterns of pain from approximately 8500 patients reported in the literature. Another way of analyzing these data is shown in Figure 3: some pain is perceived in the second division in over $44 \%$ of patients, in the third division in $35 \%$ of patients, and in the first division in $19 \%$ of patients. Thus the malar area is the most likely to be involved in the patient with tic. This pain syndrome can and does occur, however, in every area of the face and anterior scalp, and all combinations of painful sites have been reported $(2,24)$.

Tic douloureux is usually an intermittent disease. Many patients report intervals of months or even years between flurries of attacks. Recurrences are almost always in the same area of the face, but the pain may spread to new areas in subsequent attacks of tic. Intervals between attacks often decrease, and episodes of severe pain occur more frequently over time. Some patients never experience remission once their disease starts. Patients often report minor jabs in their painful areas between episodes of severe pain. Emotional and physical stress appear to increase the likelihood of severe pain in the patient who has tic, but no evidence has been found to indicate that stress plays a causative role in this disease.

\section{DIFFERENTIAL DIAGNOSIS}

Tic douloureux must be distinguished from other types of facial pain. No diagnostic studies can confirm the presence of tic douloureux. Trigeminal tic should be discriminated

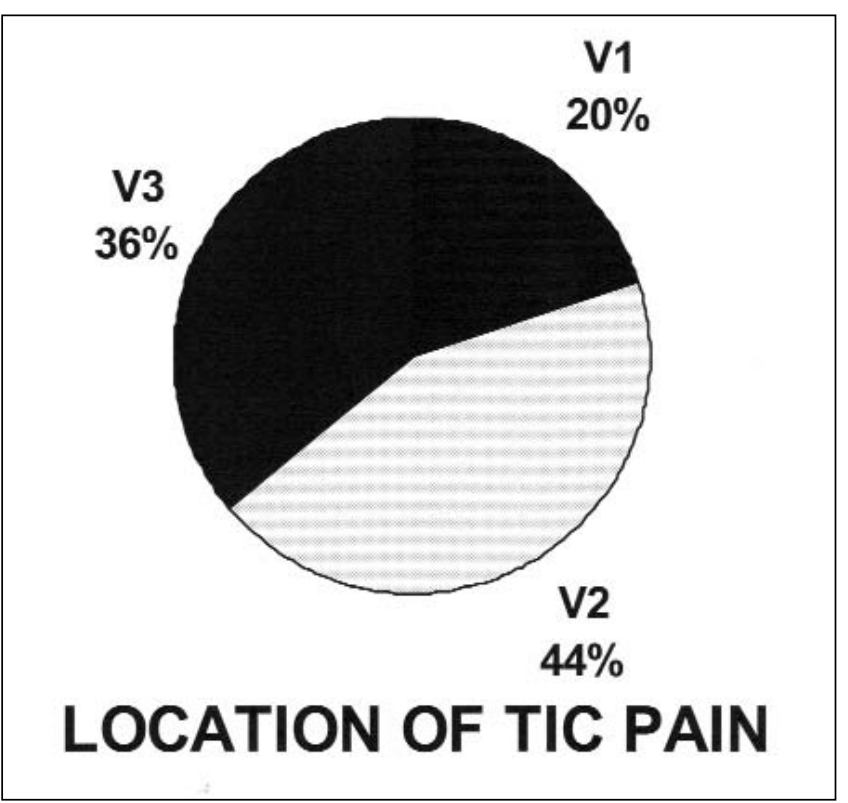

Figure 3) Trigeminal divisions involved in tic douloureux

from a similar syndrome occurring in one of the other cranial sensory nerves. This is readily accomplished by obtaining a careful history from the patient and by ascertaining the exact triggering stimulus and site of pain during the physical examination.

Atypical facial pain syndromes are the most important because these are much less likely to respond to either medicines or surgery, yet they have some affinities to tic. The atypical neuralgias form a diverse group of facial pains. Some are not restricted to a specific cranial nerve distribution; they can be bilateral, intraoral or facial, and can extend into the neck. The pain does not interfere with daily life as much as tic. They are continuous, usually with little or no sensory deficit; are not triggered; and often occur in patients with significant psychopathology. The likelihood of establishing an etiology for this type of atypical facial pain is small. Clinical studies have shown that the textbook distinctions among atypical facial pain, vascular headaches and muscle tension headaches are not respected by all patients that is, the borders of every syndrome are indistinct. Table 1 presents the characteristics of the common neurological facial pain syndromes.

Atypical facial pain may be either unilateral or bilateral. It is more common in women than in men. The bilateral variant is also known as 'burning mouth syndrome'; it has no discernable etiology and does not often respond to treatments. It is often associated with an underlying depression. Unilateral atypical facial pain can be due to overt nerve injury or an occult process such as infection or neoplasm that encroaches upon the nerve. Hence, patients with unilateral facial numbness and the complaint of pain need a thorough diagnostic evaluation. However, no explanation for the pain is found in the majority of patients. Symptomatic therapy with analgesics, anticonvulsants and antidepressants 
TABLE 2

Medications for the treatment of tic douloureux

\begin{tabular}{|c|c|c|c|c|}
\hline Generic name & $\begin{array}{c}\text { Brand name } \\
\text { (manufacturer, country) }\end{array}$ & Pill dose (mg) & Usual daily dose (mg) & Common side effects \\
\hline Phenytoin & $\begin{array}{c}\text { Epanutin (Parke-Davis, Switzerland), } \\
\text { Dilantin (Parke-Davis, Canada) }\end{array}$ & 100 & $300-500$ & $\begin{array}{c}\text { Nausea, dizziness, } \\
\text { somnolence, ataxia, dermatitis }\end{array}$ \\
\hline Carbamazepine & Tegretol (Novartis, Canada) & $100,200,300^{*}, 400$ & $600-1600$ & $\begin{array}{c}\text { Nausea, dizziness, somnolence, } \\
\text { hepatic and hematopoietic } \\
\text { dysfunction }\end{array}$ \\
\hline Oxcarbazepine $^{\dagger}$ & Trileptal (Novartis, Switzerland) & $100,300,600$ & $600-2400$ & Nausea, dizzyness, somnolence \\
\hline Baclofen & Lioresal (Novartis, Canada) & 10,20 & $40-80$ & $\begin{array}{c}\text { Nausea, dizziness, confusion, } \\
\text { drowsiness }\end{array}$ \\
\hline Gabapentin & Neurontin (Parke-Davis, Canada) & $100,300,600,800$ & $1200-4800$ & Dizziness, nausea \\
\hline Lamotrigine & Lamictal (Glaxo Wellcome, Canada) & $25,50 *, 100 *, 150,200$ & $100-500$ & Somnolence \\
\hline
\end{tabular}

${ }^{*}$ Dose not available in Canada; ${ }^{+}$Drug not available in Canada

may be helpful. Psychological therapies are often beneficial as well.

The pain of temporomandibular joint dysfunction and that of myofascial pain syndromes of the muscles of mastication are other diagnostic groups that must be distinguished from tic douloureux. The lateral location of the pain, local tenderness of the muscles and joint, continuous but fluctuating rather than intermittent nature of the pain, and absence of a trigger zone all permit easy differential diagnosis.

Myofascial pains that involve the muscles of mastication and the temporomandibular joint can be difficult to differentiate from each other but should not be confused with tic douloureux. These syndromes consist of pain that is predominantly in the lateral face, described as aching, burning or cramping, often associated with using the jaw or its muscles. There is tenderness to palpation of the involved muscles, and the pain may radiate into the scalp or neck.

Cluster headache and facial pains are a confusing group of pain syndromes. These are usually intermittent burning or throbbing dysesthetic pains that occur in grouped clear-cut episodes and are frequently associated with autonomic signs such as lachrymation, rhinitis, facial sweating and facial flushing. Most common in men, the attacks can be random or clustered and have numerous variants that are often described by an eponym. There are at least half a dozen other well-characterized facial pain syndromes that combine vascular and neuropathic features. Postherpetic neuralgia follows an outbreak of herpes zoster, and the diagnosis is usually obvious from the history.

Local pathological processes in the paranasal sinuses, jaws, teeth, pharynx or base of the skull can lead to facial pain. It is usually constant and is described as aching, throbbing or burning, but rarely as shock-like. The pain is not triggered by non-noxious stimulation remote from the painful area. If a branch of the nerve is involved in the disease, sensory loss can occur. Physical examination and the appropriate diagnostic studies usually reveal the pathology.
Modern imaging studies have dramatically facilitated the diagnoses of occult lesions of the face and skull.

\section{IMAGING STUDIES}

The only imaging study useful in the suspected diagnosis of tic douloureux is a magnetic resonance imaging (MRI) scan, especially if magnetic resonance (MR) angiography is available. Skull x-rays and computed tomography scan are rarely warranted. The MRI also establishes the diagnosis of multiple sclerosis or a mass lesion. Routine MRI scans cannot resolve the anatomical relationships between cranial nerves and adjacent blood vessels, but the addition of MR angiography and new analytic algorithms may increase the power of this technology so that the presence of a compressing artery can be discerned preoperatively. Until more sophisticated imaging strategies are widely disseminated, the primary role of an MRI scan is to rule out a rare but surgically treatable lesion other than an impinging blood vessel.

\section{PHARMACOLOGICAL TREATMENT}

The initial treatment of tic douloureux should be pharmacological, but when drugs fail, surgical therapy is indicated. The mainstays of the pharmacological management of tic douloureux are the anticonvulsant medications carbamazepine and gabapentin (Table 2). Baclofen, oxcarbazepine, phenytoin and lamotrigine can also be helpful. Other drugs are sometimes useful but are not indicated as primary medication. Scattered reports of the efficacy of other anticonvulsants have appeared but have not been corroborated with appropriately controlled studies. No alternative medicine strategies have been shown to be useful from the patient's perspective.

\section{Carbamazepine}

Carbamazepine (Tegretol; Novartis, Canada) has been the most likely drug to control tic douloureux; approximately two-thirds of patients report symptomatic improvement. This drug should be started on a daily dose of $100 \mathrm{mg}$ (one- 
half tablet); the dose is increased by increments of $100 \mathrm{mg}$ every two days until a daily dose of $600 \mathrm{mg}$ is reached. Of course, if pain relief occurs on a lower dosage, the amount taken should not be increased. After one week at a dose of $600 \mathrm{mg} /$ day, if there is no pain relief, the daily dose should be increased to $800 \mathrm{mg}$ for one week. This process can be repeated until a total daily dose of $1800 \mathrm{mg}$ is reached. Higher doses have not been more effective. Carbamazepine should be administered at least every $8 \mathrm{~h}$ to maintain stable blood levels. A sustained release preparation is available that reduces frequency of administration and helps to maintain a steady plasma level of the drug.

Carbamazepine is often a gastric irritant and should be taken with food or fluid. Some patients develop central nervous system side effects that render the drug intolerable. A small incidence of hematosuppression and liver dysfunction has been reported. Because of the gravity of this complication, a complete blood count should be obtained monthly for the first year and quarterly thereafter. Most patients have some lowering of their white blood cell count; it is not necessary to stop taking this drug unless the white blood cell concentration falls below 3500 cells $/ \mathrm{mm}^{3}$. The strategy for the use of this drug is to increase the dose until either pain relief or toxicity occurs. Serum levels have not been reliable predictors of either pain relief or toxicity. Because about $25 \%$ of patients find the side effects of carbamazepine unacceptable, successful relief of symptoms without unacceptable side effects occurs in about $50 \%$ of new patients. Carbamazepine interacts with many other drugs, and this must be carefully monitored (25).

\section{Oxcarbazepine}

Oxcarbazepine is a derivative of carbamazepine and was recently introduced as an antiepileptic. There are a few reports of its use in tic, but there are no controlled studies. There are no data on the desired serum levels. Side effects seem less common than with carbamazepine. Doses of 600 to $2400 \mathrm{mg}$ daily have been described. As with its parent drug, interactions with other medications are common with oxcarbazepine. If this drug proves to have fewer side effects, it could replace carbamazepine as the treatment of choice.

\section{Phenytoin}

Phenytoin (Dilantin; Parke-Davis, Canada) has the longest history of drugs used to treat tic. Approximately $25 \%$ of patients obtain satisfactory pain relief with serum levels of 15 to $25 \mu \mathrm{g} / \mathrm{mL}$. The goal is to maintain adequate serum levels for three weeks; if no relief of pain is seen, the drug is then discontinued. Higher doses only lead to toxicity. The usual requirement for reaching the optimal serum level is 300 to $400 \mathrm{mg}$ /day given in two divided doses. If the patient is having severe pain, it is helpful to give a $1000 \mathrm{mg}$ loading dose to achieve rapid blood levels. Central nervous system side effects are usually related to toxic serum levels, but some patients cannot tolerate even low levels of this drug. Toxic symptoms resemble excessive alcohol effects.

\section{Gabapentin}

Gabapentin is a relatively recently developed anticonvulsant that is often effective for tic douloureux. The initial dose should be $100 \mathrm{mg}$ for three days; if this dose is tolerated, it can be immediately increased to $300 \mathrm{mg}$ qid. If this dose is tolerated after one week, it can be increased gradually to a maximum of $4800 \mathrm{mg} / \mathrm{day}$. This drug is much more expensive than other anticonvulsants, and good clinical trials are not yet available. Side effects are much less common than with other anticonvulsants, but some patients have an idiosyncratic reaction at very low doses that do not provide therapeutic benefit. As with other anticonvulsants, the common side effects are drowsiness, slurred speech and incoordination.

\section{Other drugs}

Baclofen (Lioresal; Novartis, Canada) is a drug that was developed for the treatment of spasticity but was found by Fromm and colleagues (26) to be effective in some patients with tic douloureux. This drug has not had as much success in Seattle as was reported from Pittsburgh. It must be started gradually. The initial dose is 5 or $10 \mathrm{mg}$, and it is increased by 5 or $10 \mathrm{mg}$ every two days until either pain relief or toxicity occurs. The maximum dose is $80 \mathrm{mg} /$ day. The drug should be tapered and not suddenly discontinued, especially in elderly patients; acute psychosis can occur with abrupt cessation. Lamotrigine has also been shown to be effective in a small trial (27).

\section{Nerve blocks}

Injecting local anesthetic into the trigger area or the region of pain temporarily stops tic douloureux. Rarely, the patient reports pain relief that outlasts the expected duration of the local anesthetic. Local anesthesia with a few millilitres of a long-acting agent such as bupivacaine can provide a patient with some peace while adequate serum levels of an anticonvulsant are being obtained or planned surgery is undertaken. For a patient with continuous excruciating tic, a nerve block may provide an opportunity for hydration, nutrition and oral hygiene.

Alcohol blocks of the peripheral branches of the trigeminal nerve or of the gasserian ganglion have a long history in the management of tic douloureux (24). Experienced operators have reported good results, but in most physicians' hands the long term outcome is not favourable. Alcohol blocks rarely last longer than one year. Repeated blocks have a much lower success rate and increased morbidity. The amount of hypalgesia and anesthesia has been difficult to control except in the hands of experts $(28,29)$. Alcohol blocks are less satisfactory than gangliolysis and are no longer standard therapy when modern technology is available. When an experienced practitioner of gangliolysis or stereotactic radiosurgery is not available, an alcohol block might be the best alternative.

\section{Dental procedures}

Physicians, dentists and patients often believe that something must be wrong where it hurts, and this belief has led to 
TABLE 3

Neurosurgical procedures for the treatment of tic douloureux

Peripheral neurectomy

Peripheral radiofrequency or cryoprobe lesions

Gangliolysis (radiofrequency, mechanical or glycerol)

Compression or decompression of the gasserian ganglion

Subtemporal retrogasserian neurotomy

Suboccipital retrogasserian neurotomy

Trigeminal tractotomy

Microvascular decompression of the trigeminal root

Stereotactic radiosurgery

the extraction of numerous healthy teeth and to extensive endodontic procedures. Tic douloureux perceived in the nose never results in surgical extirpation of that structure, whereas tic in the teeth often results in extraction. This is a commentary on the socially determined propriety of therapies, not on an understanding of pathophysiology. Similarly, surgical procedures on the paranasal sinuses are ineffective. A focus of infection in the teeth or jaw can exacerbate the frequency of tic pain and should be treated. However, such treatments do not eliminate the pain of tic douloureux.

\section{Neurosurgical procedures}

The list of surgical treatments for tic douloureux extends from the peripheral nerve to the brain stem. Table 3 lists the commonly used surgical procedures. No surgical procedure is warranted unless pharmacological therapy has failed, because of either inadequate pain relief or unacceptable side effects. Currently, the three primary surgical treatments for tic douloureux are gangliolysis, microvascular decompression of the trigeminal nerve by a suboccipital craniectomy and stereotactic radiosurgery with the gamma knife $(6,30-$ 32). No properly conducted clinical trials have compared surgical options.

Gangliolysis: Gangliolysis is the most recent development in the long history of destructive surgical procedures for tic douloureux (33). The goal is to produce the least neurological deficit that will control the patient's pain. Gangliolysis is performed by placing a needle through the cheek and into the foramen ovale and then into the rootlets behind the gasserian ganglion. The placement of the needle is confirmed by roentgenography or fluoroscopy. Three methods are used for making a lesion: a thermally controlled radiofrequency current to heat and destroy anatomically selected portions of the posterior rootlets (34); injection of glycerol into the cistern of the trigeminal ganglion (35); and inflation of a balloon in Meckel's cave (36). Gangliolysis offers an $80 \%$ chance of one year of pain relief and a $60 \%$ chance of five years of success; the complication rate is less than $0.5 \%$ (37). Gangliolysis is particularly useful in the debilitated or elderly patient who is at increased risk from a major surgical procedure. Surgeons seem to report the best results with the type of gangliolysis that they prefer to use.
In my experience, radiofrequency gangliolysis produces the best long term results; however, patient cooperation is required. The advantage of a balloon gangliolysis is that it is done under general anesthesia and requires no patient cooperation; I have had less favourable long term results with it. Microvascular decompression: The popularity of microvascular decompression stems from the studies of Jannetta (6). It is performed under general anesthesia after a suboccipital craniectomy using a microscope to visualize the trigeminal nerve as it leaves the pons. Compression by a vascular structure is relieved by repositioning the offending artery or by coagulating and dividing a vein. This operation offers about an 85\% long term success rate and does not lead to any sensory loss. The morbidity rate is about $5 \%$ and the mortality rate $0.5 \%$. It is the only surgical procedure that produces no sensory loss and aims directly at the cause of the pain. It has the best long term success rate of any of the available surgical treatments. Frail, elderly patients may not be the best candidates for this operation because of the risks inherent in a major surgical procedure. Repeated observations by neurosurgeons have confirmed the relationship of a cross-compressing vessel to the region of the patient's facial pain. What is not understood is why the identical pain may recur 10 or 20 years later. Re-exploration of the nerve only occasionally reveals a new cross-compression; in most such cases a partial rhizotomy is warranted.

Retrogasserian neurotomy: Sectioning the trigeminal root between the gasserian ganglion and the pons was the standard intracranial procedure for the first half of the 20th century $(37,38)$. This operation is still indicated when no compression of the trigeminal nerve is discovered during suboccipital craniectomy or if microvascular decompression fails to provide long term pain relief. It is wisest to perform a partial rhizotomy because this lessens the probability of inducing anesthesia dolorosa and reduces the morbidity of a totally numb face.

Peripheral neurectomy: Peripheral neurectomy is indicated only when gangliolysis has failed and the patient cannot or will not tolerate a suboccipital craniectomy with microvascular decompression. It produces dense numbness and rarely provides more than a year of pain relief. Repeat avulsions of peripheral branches of the trigeminal nerve are even less likely to succeed.

Trigeminal tractotomy: Sectioning of the descending trigeminal tract in the medulla produces isolated loss of pain and temperature sensation in the ipsilateral face and pharynx. The operation is indicated when rhizotomy has failed to alleviate the patient's pain and when all other measures have failed. It is carried out through a small suboccipital craniectomy and laminectomy of $\mathrm{C} 1$ and $\mathrm{C} 2$. The surgical morbidity rate is high if the surgeon is inexperienced, and does not use both anatomical and physiological localization techniques. This operation also can be used for tic pain that involves the territories of the nervus intermedius and glossopharyngeal and vagal nerves. Kunc (39) showed that the pain and temperature fibres for all three of the nerves travel in the medial aspect of the descending trigeminal tract. 
Stereotactic radiosurgery: Using either a linear accelerator or the gamma knife, it is possible to place a radiosurgical lesion in the trigeminal root without damaging surrounding brain or vascular structures $(31,32)$. A recent report indicates that over 2800 patients had been treated with the gamma knife throughout the world by the end of 1998, and this number is growing exponentially as the number of gamma knife machines is rapidly increasing (32). Doses of 60 to $90 \mathrm{~Gy}$ are used in a single session; the therapeutic effects take weeks to a few months to be noted. The overwhelming majority of patients who are going to get relief from this treatment do so by six months, but there is a small number whose pain relief is delayed up to one year. As is true for all other types of treatment for tic douloureux, patients with atypical clinical features have a significantly lower chance of achieving good results (88\% compared with $56 \%$ ). The history of having had prior tic surgery also decreases the likelihood of relief by gamma knife by about $20 \%$. Less than $10 \%$ of patients develop sensory changes in their face after this treatment, and post-treatment denervation pain is very rarely observed. No other complications have thus far been reported. There are few data available on the results after five years. The short term results are as good as gangliolysis for tic douloureux, and this procedure is likely to have much more widespread use in the years to come $(31,32)$. In fact, it could completely replace percutaneous procedures of the relief of tic. Suboccipital craniectomy with microvascular decompression is the most likely surgical treatment to give long term pain relief, but this operation certainly has complications that are not seen with gamma knife radiosurgery.

\section{MULTIPLE SCLEROSIS}

Patients with multiple sclerosis can have classic trigeminal neuralgia, or they can have atypical facial pain. The number of patients with multiple sclerosis in any reported series of patients with trigeminal neuralgia is subject to various selection biases. The literature suggests that fewer than $0.5 \%$ of all patients with tic douloureux have multiple sclerosis. Females younger than 45 years are the most likely to have tic due to multiple sclerosis. This diagnosis requires the identification of nervous system lesions disseminated in both time and space. It is extremely rare for tic douloureux to be the first symptom in a patient with multiple sclerosis.

\section{NEOPLASM}

It is uncommon for a neoplasm to lead to classic trigeminal neuralgia. A constant pain associated with sensory loss, properly diagnosed as atypical facial pain, is much more common. A tiny fraction of patients with trigeminal neuralgia have a small benign neoplasm impinging on the trigeminal nerve in the subarachnoid space between the brain stem and Meckel's cave. This is usually a meningioma, neurinoma or cholesteatoma. Neoplasms intrinsic to the trigeminal nerve or those that impinge on it in Meckel's cave or further peripherally rarely produce classic trigeminal neuralgia. They are much more likely to lead to a constant aching pain that is associated with sensory loss and to other cranial nerve involvement.

\section{ANESTHESIA DOLOROSA}

Anesthesia dolorosa is the most dreaded complication of ablative lesions of the trigeminal nerve or its branches. The term refers to the complaint of pain in an anesthetic region. It is most commonly noted after surgery for atypical facial pain, but it can occur after surgery for tic douloureux or after traumatic nerve injury. It is rarely reported after ablative lesions for cancer pain in the face. The patient complains bitterly of painful numbness, which can also have burning, stabbing or aching components. The pain is not triggered and is constant. Anesthesia dolorosa is more common after lesions that totally denervate a region of the face; partial rhizotomy or careful gangliolysis are much less likely to lead to this complaint than complete rhizotomy or dense sensory loss caused by gangliolysis. No pharmacological or ablative surgical treatment has been found to be effective for anesthesia dolorosa. Electrical stimulation of the thalamus-internal capsule has produced a few good results.

\section{NERVUS INTERMEDIUS NEURALGIA}

Nervus intermedius neuralgia is an extremely rare pain syndrome in which the patient reports shock-like pains in the distribution of the nervus intermedius, which is the somatic sensory branch of cranial nerve VII. The pain is in every way similar to that of tic douloureux except for its location. Few cases have been reported since the original report by Clark and Taylor in 1909 (40,41). The patient complains of intermittent, stabbing, electric shock-like pain deep in the ear. The pain can be triggered by non-noxious stimulation of the ear canal or can follow swallowing or talking, and the patient is free of pain between attacks. Neurological deficits are absent. The syndrome is always unilateral. Some patients have reported salivation, bitter taste, tinnitus and vertigo during the pain attacks; perhaps this indicates involvement of central connections of the nervus intermedius or irritation of other components of cranial nerves VII and VIII. Rarely, patients with pain in the trigeminal distribution also have pain in the nervus intermedius territory.

Geniculate neuralgia can also be caused by herpes zoster. The patient with geniculate neuralgia usually has a vesicular eruption on the eardrum and external canal that follows the onset of the pain by one or two days. The pain is constant and burning, and is easily discriminated from the intermittent stabbing pain of nervus intermedius tic.

The medical management of nervus intermedius neuralgia is identical to that of tic douloureux (Table 2). When medications do not control the pain, a surgical procedure is warranted. It is impossible to block the nervus intermedius with local anesthetics, but they can be injected into the glossopharyngeal or trigeminal nerve to establish that these two nerves are not responsible for the pain, leaving, by subtraction, the nervus intermedius as the culprit. When medical management fails, suboccipital craniectomy with explo- 
ration of the nervus intermedius is indicated. If an offending vessel is found, it can be mobilized. If no vessel can be identified, the nervus intermedius should be sectioned. This procedure is highly likely to relieve the pain permanently, but when it does not, section of the medial aspect of the descending trigeminal tract can be performed.

\section{GLOSSOPHARYNGEAL NEURALGIA}

Glossopharyngeal neuralgia is a rare pain syndrome characterized by shock-like pains in the territory of the glossopharyngeal nerve. It is in every way similar to tic douloureux except for the distribution of the pain and the site of the triggering stimulus $(42,43)$. Patients with glossopharyngeal neuralgia have an artery compressing the nerve as it exits from the medulla and travels through the subarachnoid space to the jugular foramen (44). This syndrome also can be seen in patients with multiple sclerosis, but it is very rare. Glossopharyngeal neuralgia is characterized by excruciating shock-like pain in the region of the tonsillar fossa, pharynx or base of the tongue. It can radiate to the ear or the angle of the jaw, or into the upper lateral neck. The trigger zone is often in the same area, and patients may report that swallowing, yawning, clearing the throat or talking is the precipitating stimulus. The pain often appears to be spontaneous. Chewing or touching the face does not precipitate an attack. Glossopharyngeal neuralgia is much less common than tic douloureux. The incidence is about one in 100 .

Involvement of the glossopharyngeal nerve can be demonstrated by localizing the triggering stimulus to the pharyngeal structures that it innervates. Blocking the trigger area with local anesthetic can confirm the site of the trigger and nerve involvement. This is unsuccessful in some patients because the vagus nerve can contain the involved sensory fibres (45). The role of the glossopharyngeal nerve in the regulation of heart rate and blood pressure is thought to be the reason that some patients with glossopharyngeal neuralgia have profound cardiac arrhythmias and even asystole with the attack of pain. The presence of such phenomena guarantees that the pain syndrome involves this nerve. The diagnosis can be confirmed by the cessation of pain when the nerve is blocked at the jugular foramen or when topical anesthesia of the pharynx stops the pain.

Pharmacological management is identical to that for tic douloureux of the trigeminal nerve (Table 2). When medical management fails, suboccipital craniectomy with exploration of the glossopharyngeal nerve is indicated. If a compressing blood vessel is found it can be mobilized, and the pain usually stops without any loss of nerve function. When no structural pathology can be identified, the glossopharyngeal nerve should be sectioned. In such a case it is wise to section the upper fibres of the vagus nerve as well, because they can also be involved in the pain syndrome. When rhizotomy is unsuccessful, which happens rarely, the medial aspect of the descending tract of the trigeminal nerve can be sectioned to produce loss of pain and temperature sensation in the pharynx (40).
A percutaneous technique of glossopharyngeal neurolysis has been described, but it has not been widely used because of cardiovascular and laryngeal complications $(46,47)$.

\section{VAGAL AND SUPERIOR LARYNGEAL NEURALGIA}

The two somatic sensory branches of the vagus nerve, the auricular branch and the superior laryngeal nerve, can also be the site of a pain syndrome that resembles tic douloureux. This syndrome is exceedingly rare (48). Vagal neuralgia is characterized by paroxysms of unilateral shock-like pain in the thyroid cartilage, pyriform sinus, angle of the jaw and, rarely, in the ear. Occasionally the pain radiates into the upper thorax or up into the jaw. The trigger zone is usually in the larynx; attacks are precipitated by talking, swallowing, yawning or coughing. When other portions of the vagus nerve are involved, the patient might have hiccups, inspiratory stridor, excessive salivation or coughing. The pain is in every way typical of tic douloureux except for its location. The combination of glossopharyngeal and vagal, as well as trigeminal pain has been reported. The diagnosis is established by the history and by identifying the site of the trigger zone. Associated vagal nerve findings, as described above, also pin-point this nerve as the site of the pain. Topical anesthesia of the larynx or blockade of the superior laryngeal nerve eradicates the pain and is a useful diagnostic procedure (29).

The pharmacological treatment of vagal neuralgia is identical to that of tic douloureux (Table 2). When medications do not control the pain, suboccipital craniectomy with decompression of the upper fibres of the vagus nerve is warranted. If no lesion is seen, the upper vagal and glossopharyngeal nerves should be sectioned. This procedure is usually successful, but, when it is not, section of the medial portion of the descending trigeminal tract can be beneficial (40).

\section{CONCLUSIONS}

Tic douloureux can be accurately diagnosed on the basis of the patient's history. The absence of neurological abnormalities and the presence of a trigger area make the physical examination confirmatory. Imaging studies are characterized by the absence of pathology and are indicated before a surgical procedure. Medical management is successful in about one-half of patients. Several excellent surgical options are available when medications fail. Better treatments are dependent upon improved understanding of the mechanisms underlying this terrible pain.

\section{REFERENCES}

1. Loeser JD. Tic douloureux and atypical facial pain. In: Wall PD, Melzack R, eds. Textbook of Pain. London: Churchill Livingstone, 1984:426-34.

2. Stookey B, Ransohoff J. Trigeminal Neuralgia. Its History and Treatment. Springfield: Charles C Thomas, 1959.

3. Walker AE. A History of Neurological Surgery. Springfield: Charles C Thomas, 1959.

4. Rovitt RL. Trigeminal Neuralgia. Baltimore: Williams and Wilkins, 1990. 
5. Dandy WE. Concerning the cause of trigeminal neuralgia. Am J Surg 1934;204:447-55.

6. Jannetta PJ. Microsurgical approach to the trigeminal nerve for tic douloureux. Prog Neurosurg 1976;7:180-200.

7. Lazar ML, Kirkpatrick JB. Trigeminal neuralgia and multiple sclerosis: demonstration of the plaque in an operative case. Neurosurgery 1979;5:711-7.

8. Ratner EJ, Person P, Kleinman DJ, et al. Jawbone cavities and trigeminal and atypical facial neuralgias. Oral Surg 1979;48:3-20.

9. Shaber EP, Krol EJ. Trigeminal neuralgia - a new treatment concept. Oral Surg 1980;49:286-93.

10. Bouquot JE, Christian J. Long-term effects of jawbone curettage on the pain of facial neuralgia. J Oral Maxillofac Surg 1996;53:387-97.

11. Shankland WE II. Osteocavitation lesions (Ratner bone cavities): Frequently misdiagnosed as trigeminal neuralgia: A case report. J Craniomandib Pract 1993;11:232-6.

12. Anderson LS, Black RG, Abraham J, et al. Neuronal hyperactivity in experimental trigeminal deafferentation. J Neurosurg 1971;35:444-52.

13. Black RG. Trigeminal pain. In: Crue BL, ed. Pain and Suffering. Springfield: Charles C Thomas, 1970:119-37.

14. King RB. Evidence for a central etiology of tic douloureux. J Neurosurg 1967;26(Suppl):175-80.

15. Beaver DL. Electron microscopy of the gasserian ganglion in trigeminal neuralgia. J Neurosurg 1967;26:138-50.

16. Kerr FWL. Pathology of trigeminal neuralgia: Light and electron microscopic observations. J Neurosurg 1967;26:151-6.

17. Kerr FWL. Peripheral versus central factors in trigeminal neuralgia. In: Hassler R, Walker AE, eds. Trigeminal Neuralgia. Stuttgart: Georg Thieme Verlag, 1970:180-90.

18. Kerr FWL, Miller RH. The pathology of trigeminal neuralgia. Arch Neurol 1966;15:308-19.

19. Kugelberg E, Lindblom U. The mechanism of the pain in trigeminal neuralgia. J Neurol Neurosurg Psychiatry 1959;22:36-43.

20. Turnbull IM, Black RG, Scott JW. Reflex efferent impulses in the trigeminal nerve. J Neurosurg 1961;18:746-52.

21. Calvin WH, Loeser JD, Howe JF. A neurophysiological theory for the pain mechanism of tic douloureux. Pain 1977;3:147-54.

22. Fromm GH, Chattha AS, Terrence CF, et al. Role of inhibitory mechanisms in trigeminal neuralgia. Neurology 1981;31:683-7.

23. Brzustowics RJ. Combined trigeminal and glossopharyngeal neuralgia. Neurology 1955;5:1-10

24. Harris W. An analysis of 1,433 cases of paroxysmal trigeminal neuralgia (trigeminal-tic) and the end results of gasserian alcohol injection. Brain 1940;63:209-24.

25. Spina E, Pisani F, Perucca E. Clinically significant pharmacokinetic interactions with carbamazepine. An update. Clin Pharmacokinet 1996:31:198-214

26. Fromm GH, Terrence CF, Chattha AS. Baclofen in the treatment of trigeminal neuralgia: Double-blind study and long-term follow-up. Ann Neurol 1984;15:240-4.
27. Lunardi G, Leandri M, Albano C, et al. Clinical effectiveness of lamotrigine and plasma levels in essential and symptomatic trigeminal neuralgia. Neurology 1997;48:1714-7.

28. Ecker A. Tic douloureux: Eight years after alcoholic gasserian injection. NY State J Med 1974;74:1586-92.

29. Bonica JJ. The Management of Pain. Philadelphia: Lea \& Febiger, 1953:790-7.

30. Burchiel KJ, Steege TD, Howe JF, et al. Comparison of percutaneous radiofrequency gangliolysis and microvascular decompression for the surgical management of tic douloureux. Neurosurgery 1981;9:111-9.

31. Young RF, Vermeulen SS, Grim P, et al. Gamma knife radiosurgery for treatment of trigeminal neuralgia. Neurology 1997;48:608-14.

32. Maesawa S, Ssalame C, Flickenger JC, et al. Clinical outcomes after stereotactic radiosurgery for idiopathic trigeminal neuralgia. J Neurosurg 2001;94:14-20.

33 Sweet WH, Wespic JG. Controlled thermocoagulation of trigeminal ganglion and rootlets for differential destruction of pain fibres. J Neurosurg 1974;40:143-56.

34. Menzel J, Piotrowski W, Penzholz H. Long-term results of gasserian ganglion electrocoagulation. J Neurosurg 1975;42:140-3.

35. Hakansson S. Trigeminal neuralgia treated by injection of glycerol into the trigeminal cistern. Neurosurgery 1981;9:638-46.

36. Brown JA, Gouda JJ. Percutaneous balloon compression of the trigeminal nerve. Neurosurg Clin N Am 1997;8:53-62.

37. Fraioli B, Esposito V, Guidetti B, et al. Treatment of trigeminal neuralgia by thermocoagulation, glycerolization, and percutaneous compression of the gasserian ganglion and/or retrogasserian rootlets: Long-term results and therapeutic protocol. Neurosurgery $1989 ; 24: 239-45$.

38. Ruge DR, Brochner R, Davis L. A study of the treatment of 637 patients with trigeminal neuralgia. J Neurosurg 1958;15:528-36.

39. Kunc $Z$. Treatment of essential neuralgia of the ninth nerve by selective tractotomy. J Neurosurg 1965:23:494-500.

40. Clark LP, Taylor AS. True tic douloureux of the sensory filaments of the facial nerve. JAMA 1909;53:2144-6.

41. Furlow LT. Tic douloureux of the nervus intermedius. JAMA 1942;119:255-9.

42. Peet MM. Glossopharyngeal neuralgia. Ann Surg 1935;101:256-68.

43. Rushton JG, Stevens JC, Miller RH. Glossopharyngeal (vagoglossopharyngeal) neuralgia. Arch Neurol 1981;38:201-5.

44. Laha RK, Jannetta PJ. Glossopharyngeal neuralgia. J Neurosurg 1977;47:316-20.

45. Robson JT, Bonica JJ. The vagus nerve in surgical consideration of glossopharyngeal neuralgia. J Neurosurg 1950;7:482-91.

46. Tew JM Jr. Treatment of pain of glossopharyngeal and vagus nerves by percutaneous rhizotomy. In: Youmans J, ed. Neurological Surgery. Philadelphia: WB Saunders, 1982:3609-12.

47. Ori G, Salar G, Giron, GP. Cardiovascular and cerebral complications during glossopharyngeal nerve thermocoagulation. Anesthesia 1985;40:433-7.

48. Chawla JC, Falconer MA. Glossopharyngeal and vagal neuralgia. Br Med J 1967;2:529-31. 


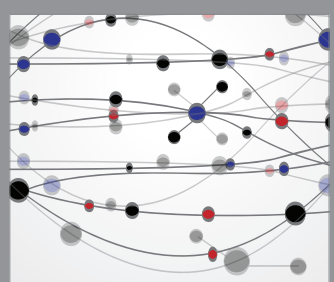

The Scientific World Journal
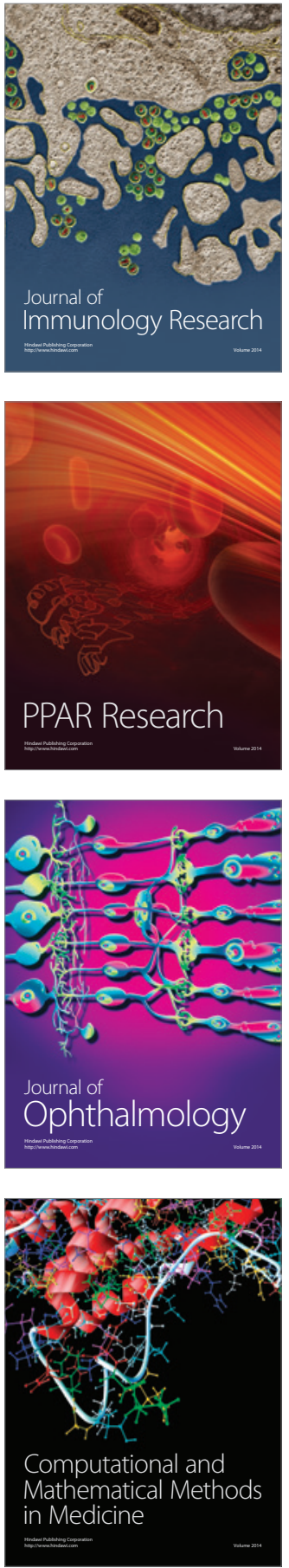

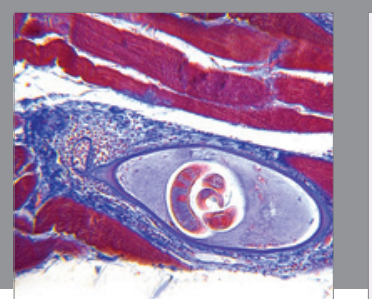

Gastroenterology Research and Practice

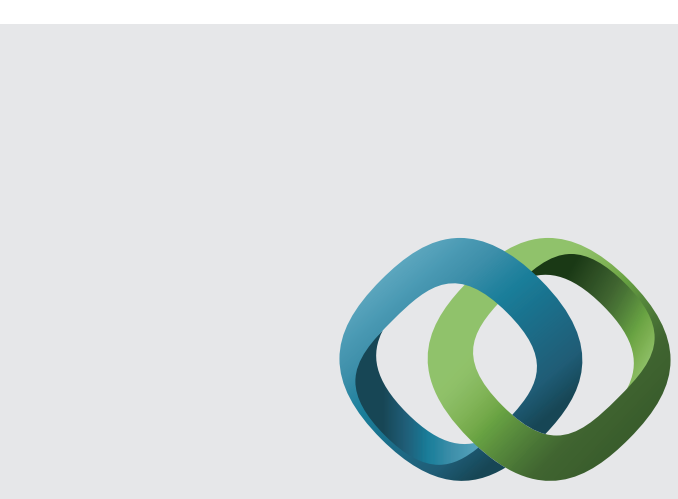

\section{Hindawi}

Submit your manuscripts at

http://www.hindawi.com
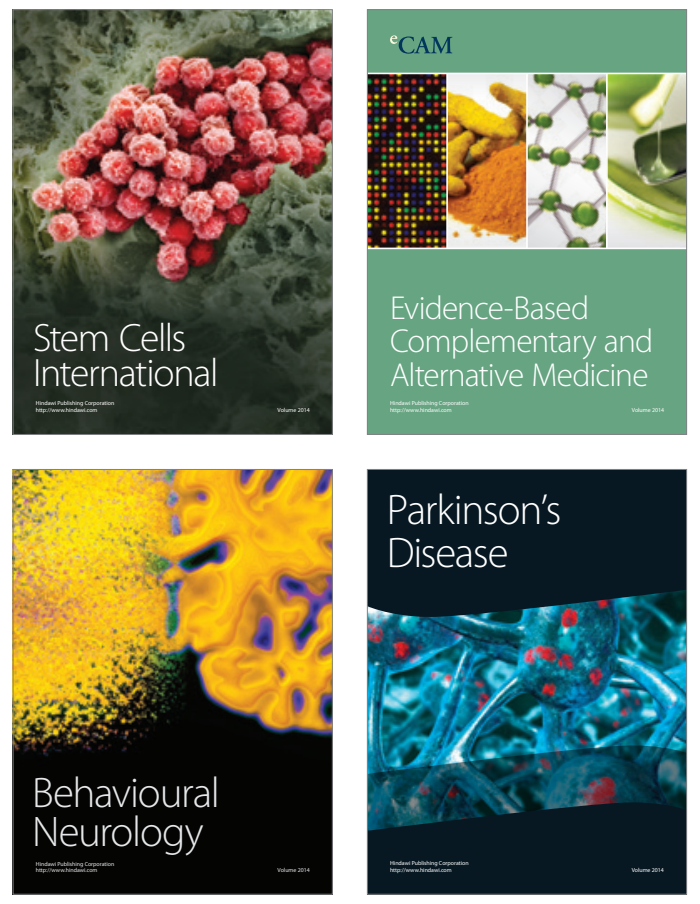
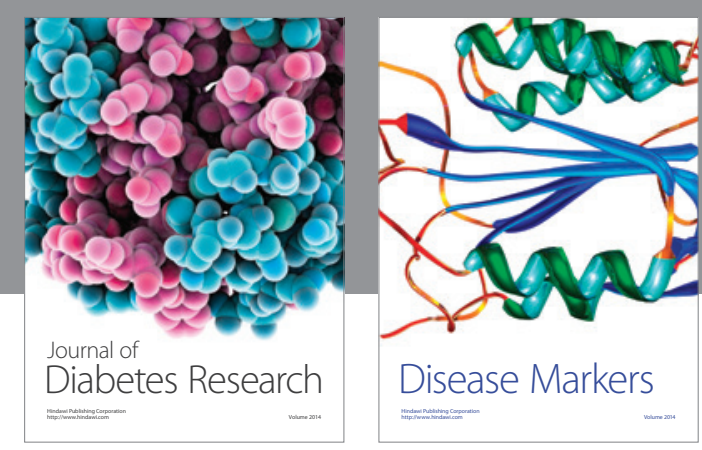

Disease Markers
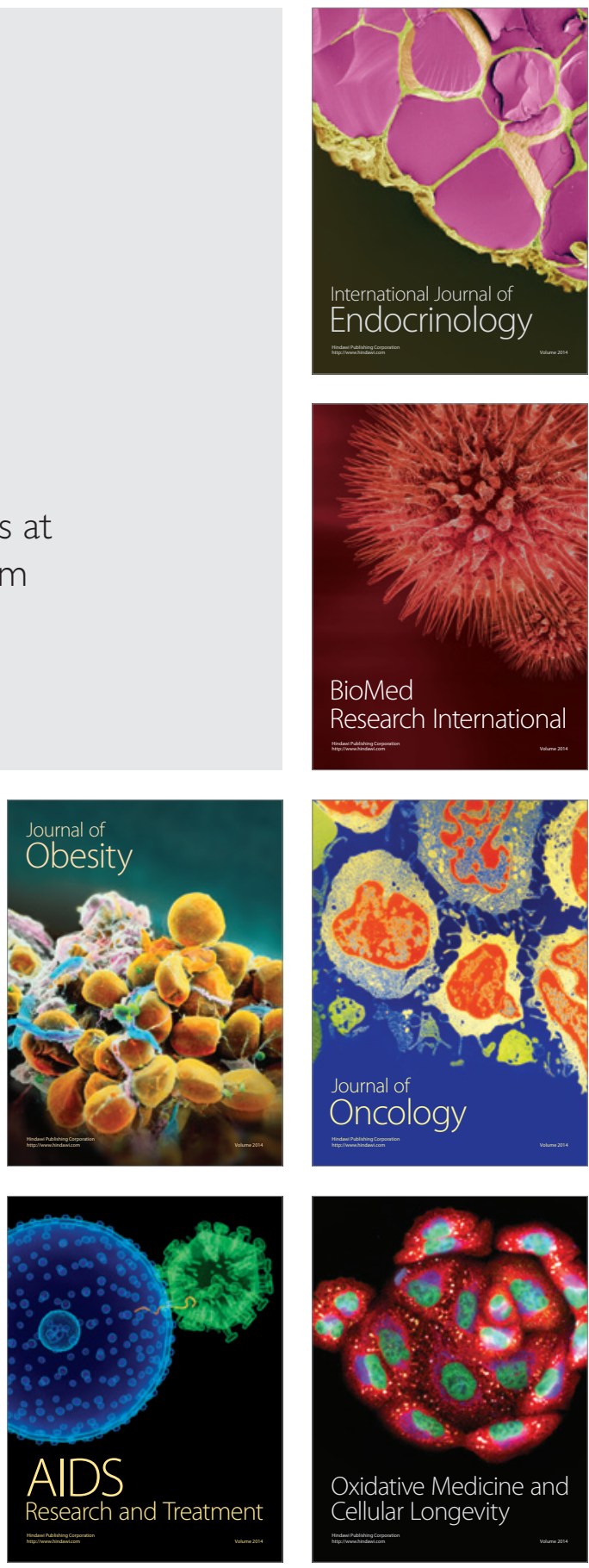